\title{
Mechanical Properties Analysis for the Connection Thread of Centralizer
}

\author{
Yin Sai Guo ${ }^{1, a^{*}}$, Yi Zhang ${ }^{1, b}$ \\ ${ }^{1}$ School of Mechanical Engineering, Xijing University, Xi'an710123, China; \\ $a^{*}$ guoysxj@163.com, 'bhangyjd@163.com
}

Keywords: Connecting thread, simulation experiment, mechanical properties analysis, ANSYS.

\begin{abstract}
Based on the theory of contact non-linear finite element analysis, the analysis for stress and strain of the connection thread of centralizer is carried out by ANSYS software. The structural weak part was identified. The method of simulation experiment is adopted. The simulation results are compared through changing the some parameters of thread structure to obtain the relation between parameters and the stress and strain of the connection thread. In order to improve the mechanical properties of weak part of connecting thread about the centralizer, some parameters which produced larger influence on its mechanical properties were selected. It provides a theoretical analysis method for improving the mechanical properties.
\end{abstract}

\section{Introduction}

Centralizer as an important kind of cementing tool is installed on the external oil coring drilling tools [1] [2]. It can improve the stability and prevent deviation when the outer cylinder and drill are working. As the working condition of oil coring drilling tools is very bad and the stress situation is very complicated, the damage of centralizer outside tube is quite serious, and most failure parts of the tube are on the connection thread. Select the reasonable structure parameters of the connection thread of centralizer to improve the mechanical properties of connection thread, which has important research significance and application value [3] [4].

\section{Finite Element Analysis}

As the helix angle of the connection thread of centralizer will have little impact on the load distribution along the thread, it can be regarded as axi-symmetric structure in processing [5]. The model can be simplified as a two-dimensional model for the simulation analysis. The thread structure of centralizer is a special kind of non-standard trapezoidal thread. On the basis of thread structure CAD drawings, accurately calculate the coordinates of each point in the model, write the corresponding APDL language, and then establish the two-dimension geometry model of a connection thread, as is shown in figure 1.

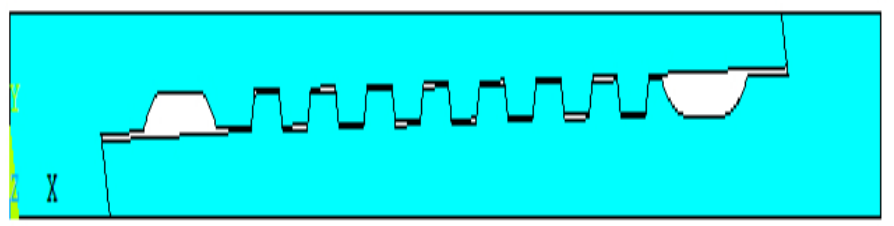

Fig. 1 Geometry model of connecting thread

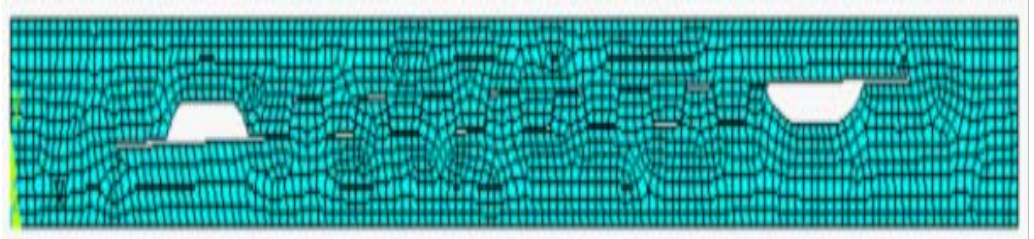

Fig. 2 Finite element model of connecting thread 
The material of the centralizer is 45CrNiMoVA, which is isotropy elastic-plastic material. Its modulus of elasticity is 2.14 x $105 \mathrm{Mpa}$; poisson's ratio is 0.29 ; the material yield limit is $1330 \mathrm{Mpa}$; material friction coefficient is 0.1 . The unit type is 8 node plane axisymmetric element PLANE82, CONTA171 (unit type reference number). The grid of the model is smartly divided. The length of unit is $1 \mathrm{~mm}$; node number is 5944; unit number is 5944 . The finite element model of connection thread is shown in figure 2.

The thread connector bears the $10 \mathrm{KN} / \mathrm{M}$ work torque and $70 \mathrm{KN}$ axial load pressure. The above two kinds of loads are converted to uniformly distributed loads on the finite element model by using the method of calculation and analysis. Fixed displacement constraints are imposed on the centreleft end of the model and distributed loads calculated are applied on the right end of the model in ANSYS software. Contact pairs are set. There are 2 contact pairs on the shoulder, 15 contact pairs on the thread interface. There are a total of 17 contact pairs. The loads applied of the finite element model are shown in figure 3 .

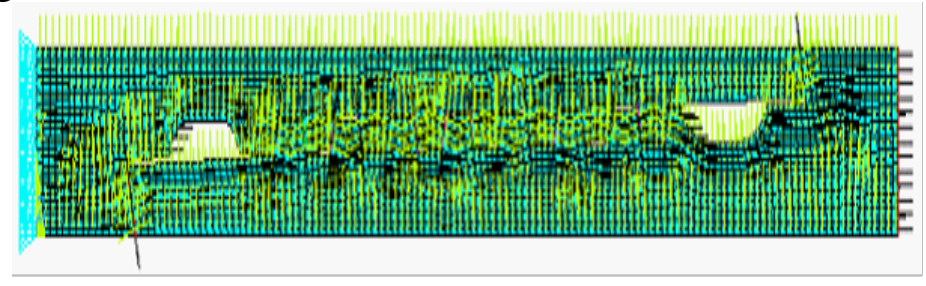

Fig. 3 Diagram about loads applied

The nonlinear contact finite element analysis was carried out on the connecting thread structure by using ANSYS [6]. The displacement and Von Mises stress contour map of the threaded connector are shown in figure 4 and 5.

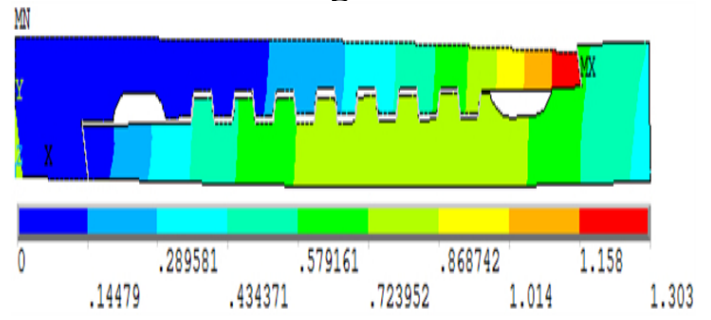

Fig. 4 Resultant displacement contour map

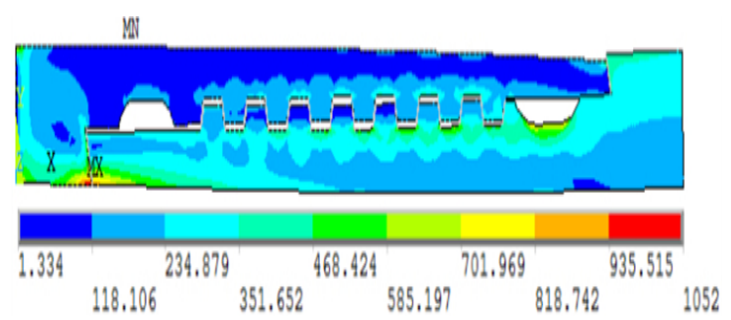

Fig. 5 Von Mises stress contour map

The biggest resultant displacement appears at the right end the female thread connector (right shoulder area) and the numerical value is $1.303 \mathrm{~mm}$, which is shown in figure 4 . From the overall deformation situation, the deformation of the male thread connector has slightly greater than the female connector. The maximum equivalent stress is $1052 \mathrm{Mpa}$, appearing in the left shoulder with MX area figure 5, which is mainly caused by the stress concentration. From the stress of the overall thread connector, Compared with other parts of the thread, the equivalent stress of thread root part is larger, and it is more likely to occur fracture failure phenomenon at the right end of the first thread.

In the case of the known load stress, the deformation and stress of connector is relatively large, as can be seen from the figure 4 and 5.So its mechanical performance (seal and bearing performance was poor. In the poor working conditions, the failure probability of thread connector is larger. The instructions from the side, the design defects exist in the original thread structure. It is necessary to optimize the structural parameters of the original thread, select some parameters larger effect on the mechanical properties.

\section{Parameter Analysis for the Infrastructure of the Thread}

The method of simulation experiment is adopted [7] [8]. The simulation results are compared through changing the some parameters of thread structure to obtain the relation between parameters and the stress and strain of the connection thread.

Mainly the following seven parameters were changed: teeth number, teeth angle, teeth height, thread pitch, thread taper, left and right basal spacing and thread diameter. As the thread connector 
is a special kind of non-standard structure of trapezoidal thread, the above values of seven parameters change under the premise of the machining process of thread structure.

From the above finite element analysis results, we can know that the maximum displacement of the original thread connector is $1.303 \mathrm{~mm}$ and the maximum equivalent stress is $1052 \mathrm{Mpa}$. The foundation structure parameters of the original thread are shown in table 1.

Table 1 Foundation structural parameters of original thread

\begin{tabular}{ccccc}
\hline Teeth number & Teeth angle & Teeth height & Thread taper & Thread pitch \\
\hline 7 & 270 & 2.24 & $1: 32$ & 8.466 \\
\hline
\end{tabular}

Table 2 The simulation results comparison about the change of thread number

\begin{tabular}{ccccc}
\hline Parameter value & 6 & 8 & 9 & 10 \\
\hline Maximum displacement & 1.151 & 1.684 & 2.27 & 2.849 \\
Maximum equivalent stress & 1010 & 1198 & 1471 & 1678 \\
\hline
\end{tabular}

Table 3 The simulation results comparison about the change of thread tooth height

\begin{tabular}{ccccc}
\hline Parameter value & 1.4 & 1.8 & 2.7 & 3 \\
\hline Maximum displacement & 1.051 & 1.195 & 1.392 & 1.416 \\
Maximum equivalent stress & 914.8 & 959.4 & 1384 & 1088 \\
\hline Table 4 The simulation results comparison of about the change of thread tooth type angle \\
\hline Parameter value & 220 & 320 & 370 & 400 \\
\hline Maximum displacement & 1.223 & 0.955 & 0.9781 & 914.214 \\
\hline
\end{tabular}

Table 5 The simulation results comparison of about the change of thread taper

\begin{tabular}{ccccc}
\hline Parameter value & $1: 8$ & $1: 16$ & $1: 24$ & $1: 32$ \\
\hline Maximum displacement & 1.448 & 0.9603 & 1.441 & 1.303 \\
Maximum equivalent stress & 1637 & 882.49 & 1114 & 1052 \\
\hline Table 6 The simulation results comparison of about the change of pitch & 10.466 \\
\hline Parameter value & 7.466 & 8.466 & 9.466 & 1.8 \\
Maximum displacement & 1.054 & 1.303 & 1045 \\
Maximum equivalent stress & 820.2 & 1052 & & 1562 \\
\hline
\end{tabular}


Table 7 The simulation results comparison of about the change of thread diameter

\begin{tabular}{ccccc}
\hline Variable quantity & $-2 \mathrm{~mm}$ & $-1 \mathrm{~mm}$ & $0 \mathrm{~mm}$ & $+1 \mathrm{~mm}$ \\
\hline Maximum displacement & 1.098 & 0.9299 & 1.303 & 1.202 \\
Maximum equivalent stress & 908.1 & 868.72 & 1052 & 1033 \\
\hline
\end{tabular}

Table 8 The simulation results comparison of about the change of the left and right basal spacing

\begin{tabular}{ccccc}
\hline Variable quantity & $-3 \mathrm{~mm}$ & $-2 \mathrm{~mm}$ & $-1 \mathrm{~mm}$ & $+1 \mathrm{~mm}$ \\
\hline Maximum displacement & 0.888 & 0.955 & 0.9781 & 1.453 \\
Maximum equivalent stress & 790.3 & 857.7 & 934 & 1168
\end{tabular}

Note: the involved length unit in table1-8 is mm; the equivalent stress unit is MPa. The "-" or "+"show that the new structural parameters decrease or increase compared with the original parameters in table 7 and 8 . The thread diameter changed refers to change the value of the large diameter, intermediate diameter and minor diameter of the thread at the same time in table 7.

From the table 1 to 8, the larger factors impacting mechanical properties of the thread connector (seal and bearing performance): thread taper, pitch, diameter and basal spacing.

\section{Summary}

A nonlinear finite element analysis of centralizer special connection thread is made to identify the defect of the original thread structure in ANSYS software. The method of simulation experiment is adopted. The simulation results are compared through changing the some parameters of thread structure to obtain the relation between parameters and the stress and strain of the connection thread. Some parameters which produced larger influence on its mechanical properties were selected. It provides a theoretical analysis method for improving the mechanical properties.

\section{Acknowledgment}

This study was supported by the Natural Science Foundation of Shaanxi Province (Program No. 2013JM8040), the Scientific Research Program Funded by Shaanxi Provincial Education Department (Program No. 2013JK1204) and Graduate Student Innovation Fund of Xijing University.

\section{References}

[1] Brown, R.C.A.; Hofmann, H.; Pegat, D.; Vullierme, B.; Kajetanowicz, M Distributed engineering controls for the DELPHI gas supply system, Volume 36, THIssue 1HT, Part 1, Feb. 1989 Page(s):71 - 73

[2] Shi Xiaobing, Shi Taihe. Study for a new type of drill string stabilizer link thread stress reduction zone [J]. Journal of natural gas industry, 2002, 22 (2) : 48-50.

[3] Ye Yong Shi Yongjun, WenChangFei etc. Based on the structure of the dynamic pressure lubrication spin centralizer design [J]. Oil drilling technology, 2011, 33 (4) : 121-121.

[4] Li Shuhua, Xu Jing. Large centralizer blank hot extrusion process difficulties and solutions [J]. Journal of thermal processing, 2013, (21) : 217-218.

[5] Su Jijun,Yin Kun,Guo Tongtong.Optimization of the Joint-Thread of Diamond Wire-Line 
Coring Drill Pipe[J].Journal of Jilin University,2005,35(5):677-680.

[6] Zhang Youzhen,Shi Zhijun,Tian Dongzhuang,et al.Finite element analysis and improved design for taper trapezium joint threads of high-strength drilling rod with big hole[J].Journal of China Coal Society,2010,35(7):1219-1223.

[7] Lin Tengjiao,Li Runfang,Xu Mingyu.Numerical Simulation of Elastoplastic Contact Characteristic for Drillpipe Thread Compounds with Two Steps.Machine Design and Research,2004,20(1):48-49.

[8] Deibel, F.L, Calculating residual manufacturing stresses in braze joints using ANSYS , Volume 34, THIssue 5HT, May 1987 Page(s):1214 - 1217, THElectron Devices, IEEE Transactions on. 